\title{
On models of directed path non rooted directed path graph
}

Marisa Gutierrez ${ }^{1}$, Silvia B. Tondato ${ }^{2}$

1 Conicet, Departamento de Matemática Universidad Nacional de La Plata, Calle 50 y 115 (1900) La Plata Argentina

2 Departamento de Matemática Universidad Nacional de La Plata, Calle 50 y 115 (1900) La Plata Argentina

\begin{abstract}
A directed path graph is the intersection graph of a family of directed subpaths of a directed tree. A rooted path graph is the intersection graph of a family of directed subpaths of a rooted tree. Clearly, rooted path graphs are directed path graphs. Several characterizations are known for directed path graphs: one by forbidden induced subgraphs and one by forbidden asteroids. It is an open problem to find such characterizations for rooted path graphs. With the purpose of proving knowledge in this direction, we show in this paper properties of directed path models that are not rootable for chordal graphs with any leafage and with leafage four. Therefore, we prove that for leafage four directed path graphs minimally non rooted path graphs has a unique asteroidal quadruple and can be characterized by the presence of certain type of asteroidal quadruples.
\end{abstract}

Key words. Clique trees, rooted path graphs, asteroidal quadruples.

\section{Introduction}

A graph is chordal if it contains no cycle of length at least four as an induced subgraph. A classical result [5] states that a graph is chordal if and only if it is the (vertex) intersection graph of a family of subtrees of a tree. It is easy to see that any interval graph is a chordal graph considering that it is the intersection graph of a family of subpaths of a path.

An asteroidal triple in a graph $G$ is a set of three non- adjacent vertices such that for any two of them there exists a path between them that does not intersect the neighborhood of the third.

Lekkerkerler and Boland [8] proved that a chordal graph is an interval graph if and only if it contains no asteroidal triple. As byproduct, they found a characterization of interval graphs by forbidden induced subgraphs.

Another natural subclass of chordal graphs are path graphs. A graph is a path graph if it is the intersection graph of a family of subpaths of a tree. Lévêque, Maffray and Preissman [9], found the characterization of path graphs by forbidden induced subgraphs but there is still no nice characterization in terms of forbidden asteroids for this class.

Two subclasses of path graphs have been defined when the host tree is a directed graph. Firstly, a graph is a directed path graph if it is the intersection graph of a family

Send offprint requests to: 
of directed subpaths of a directed tree. Panda [12], found the characterization of directed path graphs by forbidden induced subgraphs and then Cameron, Hoáng and Lévêque [2] gave a characterization of this class in terms of forbidden asteroidal triples. Secondly, a graph is a rooted path graph if it is the intersection graph of a family of directed subpaths of a rooted tree. By definition we have the following inclusions between the different considered classes (and these inclusions are strict):

interval $\subset$ rooted path $\subset$ directed path $\subset$ path $\subset$ chordal

Characterizing rooted path graphs by forbidden induced subgraphs or forbidden asteroids are open problems. It is certainly too difficult to characterizing rooted path graphs by forbidden induced subgraphs as there are too many (families of) graphs to exclude but Cameron, Hoáng and Lévêque [1] gave a conjecture which propose a characterization of these graphs in terms of forbidden asteroids. This conjecture is in fact an attempt to characterize directed path graphs that are non rooted path graphs by forbidding particular type of asteroidal quadruples. An asteroidal quadruple in a graph $G$ is a set of four non adjacent vertices such that any three of them is an asteroidal triple. In its original form, this conjecture is incomplete as was showed in [6]. Gutierrez, Lévêque and Tondato [6] proved also that every directed path graph that is not rooted path graph has an asteroidal quadruple. But clearly, it is not a characterization. In Figure 1, we show two DV graphs containing asteroidal quadruples. One is RDV graph but the other is not.

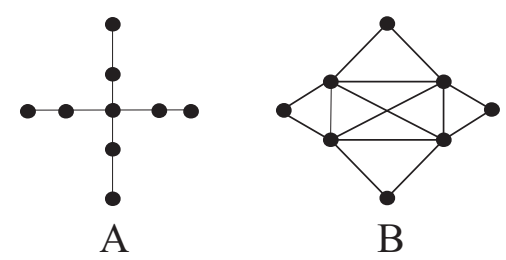

Fig. 1. The graph $\mathrm{A}$ is RDV but the graph $\mathrm{B}$ is not

As suggested by the conjecture, a characterization by forbidding particular type of asteroidal quadruples may holds.

For this purpose, we study in this paper properties of models of directed path graph non rooted directed path graph. As we said before, any model will be at least four leaves by the presence of an asteroidal quadruple [10]. We will study properties in models with any number of leaves but we will obtain special properties in case of models with four leaves. In this particular case we proved that if a graph is a directed path graph non rooted directed path graph and minimal with this property, it has an unique asteroidal quadruple. On the other hand, some paths in the graph which force the direction in any $D V$-model will be relevant. It is clear that one of this is a path of three vertices between two non adjacent. However, as was proved in [1], there are other type of paths with this property. We will prove some conditions assuring the presence of that type of paths in models with any number of leaves, and others in models with four leaves.

Formally, we will say that two non adjacent vertices of a DV graph are linked by a strong connection if for any DV-model the path between them is directed. 
Finally, we propose the following conjecture: any $D V$ non $R D V$ graph has an asteroidal quadruple with two disjoint pairs of vertices linked by a strong connections, proving it for graphs with leafage four.

The paper is organized as follows: in section 2, we give definitions and notations. In section 3, we recall and prove some properties of directed path graph non rooted directed path graphs and analyze the presence of twins and false twins. In section 4 , we study $D V$ models on those graphs in both any or four leafage. In section 5, we prove the existence of special connections for certain kind of graphs in both any or four leafage. Finally, in section 6 , we prove the conjecture in graph with leafage four.

\section{Definitions and notations}

A clique in a graph $G$ is a maximal set of pairwise adjacent vertices. Let $\boldsymbol{C}(G)$ be the set of all cliques of $G$.

The neighborhood of a vertex $x$ is the set $N(x)$ of vertices adjacent to $x$ and the closed neighborhood of $x$ is the set $N[x]=\{x\} \cup N(x)$. A vertex is simplicial if its (closed) neighborhood is a clique. Two adjacent vertices $x$ and $y$ are twins if $N[x]=N[y]$ and they are false twins if $N(x)=N(y)$.

A clique tree $T$ of a graph $G$ is a tree whose vertices are the elements of $\boldsymbol{C}(G)$ and such that for each vertex $x$ of $G$, those elements of $\boldsymbol{C}(G)$ that contain $x$ induce a subtree of $T$, which we will denote by $T_{x}$. Note that $G$ is the intersection graph of the subtrees $\left(T_{x}\right)_{x \in V(G)}$. Gavril [5] proved that a graph is chordal if and only if it has a clique tree. Clique trees are called models of the graph.

In [11], Monma and Wei introduced the notation UV, DV and RDV to refer to the classes of path graphs, directed path graphs and rooted path graphs respectively. They also prove the following clique tree characterizations for these classes. A graph is a path graph or a $U V$ graph if it admits a $U V$-model, i.e. a clique tree $T$ such that $T_{x}$ is a subpath of $T$ for every $x \in V(G)$. A graph is a directed path graph or a $D V$ graph if it admits a $D V$ model, i.e a clique tree $T$ whose edges can be directed such that $T_{x}$ is a directed subpath of $T$ for every $x \in V(G)$. A graph is a rooted path graph or a $R D V$ graph, if it admits a $R D V$-model, i.e a clique tree $T$ that can be rooted and whose edges are directed from the root toward the leaves such that $T_{x}$ is a directed subpath of $T$ for every $x \in V(G)$.

It has been proved in [4] that if $G$ is a DV graph, then any UV-model of $G$ can be directed to obtain a DV-model of $G$. We say that a DV-model $T$ of a DV graph $G$ can be rooted if $T$ can be rooted on a vertex such that it becomes a RDV-model of $G$.

Let $T$ be a clique tree. We often use capital letters to denotes the vertices of a clique tree as these vertices correspond to cliques of $G$. In order to simplify the notation, we often write $X \in T$ instead of $X \in V(T)$, and $e \in T$ instead of $e \in E(T)$. If $T^{\prime}$ is a subtree of $T$, then $G_{T^{\prime}}$ denotes the subgraph of $G$ that is induced by the vertices of $\cup_{X \in V\left(T^{\prime}\right)} X$.

If $G, G^{\prime}$ are two graphs, then $G+G^{\prime}$ we will denote the union of $G$ and $G^{\prime}$, that is the graph whose vertices are $V(G) \cup V\left(G^{\prime}\right)$ and edges are $E(G) \cup E\left(G^{\prime}\right)$. Note that if $T, T^{\prime}$ are two trees such that $\left|V(T) \cap V\left(T^{\prime}\right)\right| \leq 1$, then $T+T^{\prime}$ is a forest.

If $G$ is a graph and $V^{\prime} \subseteq V(G)$, then $G \backslash V^{\prime}$ denotes the subgraph of $G$ induced by $V(G) \backslash V^{\prime}$. If $E^{\prime} \subseteq E(G)$, then $G-E^{\prime}$ denotes the subgraph of $G$ induced by $E(G) \backslash E^{\prime}$.

Let $T$ be a tree. For $V^{\prime} \subseteq V(T)$, let $T\left[V^{\prime}\right]$ be the minimal subtree of $T$ containing $V^{\prime}$. Then for $X, Y \in V(T), T[X, Y]$ is the subpath of $T$ between $X$ and $Y$. Let $T[X, Y)=$ $T[X, Y] \backslash Y, T(X, Y]=T[X, Y] \backslash X$ and $T(X, Y)=T[X, Y] \backslash\{X, Y\}$. Note that some 
of these paths may be empty or reduced to a single vertex when $X$ and $Y$ are equal or adjacent. If $X \in V(T)$ and $e \in E(T)$ with $e=A B$ and $A \in T[X, B]$, then let $T[X, e]=T[X, B], T[X, e)=T[X, A], T(X, e]=T(X, B]$ and $T(X, e)=T(X, A]$. A vertex $X \in V(T(Y, Z))$ has a vertex crossing in $T[Y, Z]$ if $X^{\prime} \cap X^{\prime \prime} \neq \emptyset$ where $X^{\prime}$ and $X^{\prime \prime}$ are the two neighbors of $X$ in $T[Y, Z]$. If $X$ is not a leaf and $X=Y$ or $X=Z$, we say that $X$ has a vertex crossing in $T[Y, Z]$ if $X^{\prime} \cap X^{\prime \prime} \neq \emptyset$ where $X^{\prime}$ is the neighbor of $X$ in $T[Y, Z]$ and $X^{\prime \prime}$ is a neighbor of $X$ in $T$ different from $X^{\prime}$.

In a clique tree $T$, the label of an edge $A B$ of $T$ is defined as $l a b(A B)=A \cap B$. We say that $X \in V(T)$ dominates $e \in E(T)$ if $l a b(e) \subseteq X$. We say that $e^{\prime} \in E(T)$ dominates $e \in E(T)$ if $l a b(e) \subseteq l a b\left(e^{\prime}\right)$. If two edges $e, e^{\prime}$ satisfy $l a b(e)=l a b\left(e^{\prime}\right)$, we says that $e$ and $e^{\prime}$ are equivalent edges. We say that an edge $e$ satisfying a given property $P$ is maximally farthest from a vertex $C$ if there is no edge $e^{\prime}$, distinct from $e$, satisfying this property and such that $e$ is between $C$ and $e^{\prime}$.

Let $T$ be a clique tree of $G$. Let $\mathbf{D}(T)$ (or $\mathbf{D}$ for short) be the vertices of $T$ of degree at least three. Observe that if $T$ is not a path and $H$ is a leaf of $T$ then there exists $C \in T[\mathbf{D}]$ such that $T[H, C] \cap T[\mathbf{D}]=\{C\}$. In this case we say that $T[H, C]$ is a branch of $T$ incident to $C$. Let $C$ be a element of $\mathbf{D}$. The union of all the branches incident to $C$ forms a subtree of $T$ called the bouquet of $T$ incident to $C$ and denoted $\operatorname{Bouq}(C)$. Note that if $C$ is a leaf of $T[\mathbf{D}]$ then there are at least two branches in $\operatorname{Bouq}(C)$ (otherwise $\operatorname{Bouq}(C)$ can be empty or reduced to one branch).

Let $T$ be a tree, we denote by $\ln (T)$ the number of leaves of $T$. The leafage of a chordal graph $G$ is the minimum integer $\ell$ such that $G$ admits a model $T$ with $\ln (T)=\ell$. $U V$ leafage, $D V$-leafage and RDV-leafage are defined analogously with $T$ being a UV-model, DV-model and RDV-model respectively. For any DV graph we know that DV-leafage $=$ UV-leafage = leafage. The first equality is a consequence of [4], the second equality has been proved independently in [3] and [7]. Surprisingly for RDV graph the equality does not hold [6].

An asteroidal set $A$ in a graph $G$ is a set of non adjacent vertices such that for any $v \in A$ the vertices of $A \backslash\{v\}$ appears in the same connected component of $G \backslash N[v]$. Note that this definition is compatible with the definition of asteroidal triple and quadruple already given. The asteroidal number of a graph $G$ is the maximum integer $a$ such that $G$ admits an asteroidal set of cardinality $a$. If $G$ is a chordal graph containing an asteroidal set $A$ of size $k$, then in for any model $T$ of $G, T$ has at least $k$ leaves [10]. Thus the asteroidal number of a chordal graph is less or equal to its leafage.

Let $T$ be a DV-model of $G$, and $T^{\prime}$ a (non necessarily proper) subtree of $T$, we say that a vertex $H$ of $T$ is good in $T^{\prime}$ if there is no edge of $T^{\prime} \backslash H$ dominated by $H$, otherwise $H$ is bad in $T^{\prime}$. We just say that a leaf $H$ of $T$ is good (resp. bad) if it is good in $T$ (resp. bad in $T$ ). Good leaves are related to the existence of asteroidal sets by the following lemma.

Lemma 1. [6] Let $T$ be a clique tree of a graph $G$ and $V^{\prime} \subseteq V(T)$. If for every $X \in V^{\prime}$, $X$ is a leaf of $T\left[V^{\prime}\right]$ that is good in $T\left[V^{\prime}\right]$, then $G$ has an asteroidal set $A$ of size $\left|V^{\prime}\right|$ where each set $X \in V^{\prime}$ contains exactly one distinct vertex of $A$.

\section{Preliminaries results}

First, we recall some general properties of graph $D V$ non $R D V$ proved in [6].

Theorem 1. If $G$ is a $D V$ graph non $R D V$ then it has an asteroidal quadruple. 
Corollary 1. Let $G$ be a $D V$ graph non $R D V$. Then $l(G)>3$.

Second, we analyze the presence of twins and false twins. As it is natural in this kind of intersection graphs twins are not allowed in minimal forbidden subgraphs. However, false twins apperar in chordal minimal non UV graphs but not in DV minimal non RDV graphs.

Theorem 2. Let $G$ be a $D V$ graph, minimally non $R D V$. Then $G$ does not have twins nor false twins.

Proof. Let $x$ and $y$ be twins of $G$. By minimality of $G, G \backslash x$ is a $R D V$ graph and let $T^{\prime}$ be a $R D V$-model of $G \backslash x$. Observe that $C \in \boldsymbol{C}(G \backslash x)-\boldsymbol{C}(G)$ if and only if $C \cup\{x\} \in \boldsymbol{C}(G)$. Let $C_{1}^{\prime}, . ., C_{k}^{\prime}$ be the cliques of $G \backslash x$ containing $y$ and $C_{i}=C_{i}^{\prime} \cup\{x\}$ for $i=1, . ., k$. Let $T$ be a tree built replacing $C_{i}^{\prime}$ by $C_{i}$ in $T^{\prime}$. It is clear that $T$ is a $R D V$-model of $G$, a contradiction.

Let $x$ and $y$ be false twins of $G$. Clearly, $x$ and $y$ must be simplicial vertices of $G$. By minimality of $G, G \backslash x$ is a $R D V$ graph. Let $T^{\prime}$ be a $R D V$-model of $G \backslash x$ rooted on a vertex $R$. Observe that as $G$ does not have twins and it is minimally non $R D V$ then there is an edge $e=A B \in T^{\prime}$ such that $\operatorname{lab}(e)=N(y)=N(x)$. On the other hand, $N[x]$ is a clique of $G$. Then $T=T^{\prime}-e+A N[x]+N[x] B$ rooted on $R$ is a $R D V$-model of $G$, a contradiction.

\section{DV minimally non RDV graphs and their DV-models}

Clearly in a $D V$ non $R D V$ graph none of its $D V$ models is rootable. Hence we need to study deeply properties of these models; specially in case that the graph is minimal in the difference of both classes. We separate this section according to the leafage.

\subsection{Any leafage}

Lemma 2. Let $G$ be a $D V$, minimally non $R D V$ graph, $T$ a clique tree of $G, H$ a leaf of $T$ and $T[H, C]$ a branch of $T$. Then each vertex $X$ in the branch different from $H$, has $a$ vertex crossing $X$ in $T[H, C]$.

Proof. Suppose by contradiction, that $X$ does not have a vertex crossing in $T[H, C]$. Let $X^{\prime}$ and $X^{\prime \prime}$ be neighbors of $X$. Then $X^{\prime} \cap X^{\prime \prime}=\emptyset$. Let $T^{\prime}=T-E(T[H, X])$. The subgraph $T^{\prime}$ is a proper subtree of $T$. By minimality of $G, G_{T^{\prime}}$ is a $R D V$ graph. Let $T^{\prime \prime}$ be a $R D V$ model of $G_{T^{\prime}}$ rooted on a vertex $R$. Thus it is possible to build a $R D V$-model of $G$ with $T^{\prime \prime}$ and $T[H, X]$ as follows: $T^{\prime \prime}+T[H, X]$ rooted on $R$, a contradiction.

In the next lemmas we use the following notation: for $e=A B$ an edge of a branch $T[H, C], B \in T[A, C]$.

Lemma 3. Let $G$ be a $D V$, minimally non $R D V$ graph, $T$ a clique tree of $G, H$ a leaf of $T$, and $T[H, C]$ a branch of $T$. For all e edge of $T[H, C]$ :

1. $|\operatorname{lab}(e)|>1$

2. There are at least two vertices $x, y \in l a b(e)$ such that $T_{x}$ and $T_{y}$ have different end towards $C$, i.e $T_{x} \cap\left(T-E(T[H, A]) \neq T_{y} \cap(T-E(T[H, A])\right.$. 
Proof. 1. Suppose by contradiction, that $e=A B$ is an edge of $T[H, C]$ with $A \cap B=\{x\}$ for $x \in V(G)$. Let $T^{\prime}=T-E(T[H, B])$. The subgraph $T^{\prime}$ is a proper subtree of $T$. By minimality of $G, G_{T^{\prime}}$ is a $R D V$ graph. Let $T^{\prime \prime}$ be a $R D V$-model of $G_{T^{\prime}}$ rooted on a vertex $R$. Let $Z, W \in T^{\prime \prime}$ such that $T_{x}^{\prime \prime}=T^{\prime \prime}[Z, W]$ and $W \in T^{\prime \prime}[Z, R]$. Thus it is possible to build a $R D V$-model of $G$ with $T^{\prime \prime}$ and $T[H, X]$ as follows: $T^{\prime \prime}+Z A+T[A, H]$ rooted on $R$, a contradiction.

2. Suppose by contradicting, that every vertex $x$ in $l a b(e)$ satisfies $T_{x} \cap(T-E(T[H, A]))=$ $T[A, X]$ for some $X$ in $T$. Let $T^{\prime}=T-E(T[H, B])$. The subgraph $T^{\prime}$ is a proper subtree of $T$. By minimality of $G, G_{T^{\prime}}$ is a $R D V$ graph. Let $T^{\prime \prime}$ be a $R D V$-model of $G_{T^{\prime}}$ rooted on a vertex $R$. All vertices of $l a b(e)$ are twins in $G_{T^{\prime}}$ as for $x \in l a b(e), T_{x}^{\prime \prime}=T^{\prime \prime}[Z, W]$ and $W \in T^{\prime \prime}[Z, R]$. As in 1 , it is possible to build $T^{\prime \prime}+Z A+T[A, H]$ rooted on $R$ that is a $R D V$-model of $G$, a contradiction.

Lemma 4. Let $G$ be a $D V$, minimally non $R D V$ graph, $T$ a clique tree of $G$ and $X, Y$ vertices of $T$ such that all the vertices of $T(X, Y)$ has degree two in $T$ then there are not two edges in $T[X, Y]$ with the same label.

Proof. Suppose by contradiction, that $e_{1}$ and $e_{2}$ are edges of $T[X, Y]$ that have the same label. Let $e_{1}=A_{1} B_{1}$ and $e_{2}=A_{2} B_{2}$ with $A_{1} \in T\left[X, B_{1}\right]$ and $A_{2} \in T\left[X, B_{2}\right]$. Let $T^{\prime}=$ $T-E\left(T\left[A_{1}, B_{2}\right]\right)+A_{1} B_{2}$. The subgraph $T^{\prime}$ is a proper subtree of $T$. By minimality of $G$, $G_{T^{\prime}}$ is a $R D V$ graph. Let $T^{\prime \prime}$ be a $R D V$-model of $G_{T^{\prime}}$ rooted on a vertex $R$. Let $\widetilde{e}=\widetilde{A_{1}} \widetilde{B_{2}}$ be an equivalent edge of $e$ in $T^{\prime \prime}$. Clearly, $A_{1} \cap B_{2} \subseteq \widetilde{B_{2}}$ and $A_{1} \cap B_{2} \subseteq \widetilde{A_{1}}$. Thus it is possible to build a $R D V$-model of $G$ with $T^{\prime \prime}$ and $T\left(A_{1}, B_{2}\right)$ as follows: $T^{\prime \prime}-\widetilde{A_{1}} \widetilde{B_{2}}+\widetilde{A_{1}} T\left(A_{1}, B_{2}\right) \widetilde{B_{2}}$ rooted on $R$, a contradiction.

Lemma 5. Let $G$ be a $D V$, minimally non $R D V$ graph, $T$ a clique tree of $G$, $H$ a leaf of $T$, and $T[H, C]$ a branch of $T$. Every edge of the branch $T(H, C]$ has a dominated edge outside.

Proof. Suppose that none edge outside $T[H, C]$ is dominated by $e$. Take $e^{\prime}$ dominated by $e$ in $T(H, C]$ nearest $C$ (it could be $e$ ). Let $e^{\prime}=A^{\prime} B^{\prime}$ with $B^{\prime} \in T\left[A^{\prime}, C\right]$ and $T^{\prime}=$ $T-E\left(T\left[H, A^{\prime}\right]\right)$. By the election of $e^{\prime}, A^{\prime}$ is a leaf in any clique tree of $G_{T^{\prime}}$. Let $T^{\prime \prime}$ be a $R D V$-model of $G_{T^{\prime}}$ rooted on a vertex $R$. Clearly, $T^{\prime \prime}$ has only one edge equivalent to $e^{\prime}$ and this edge must be incident in $A^{\prime}$. Hence the $D V$ model of $G T^{\prime \prime}+T\left[H, A^{\prime}\right]$ can be rooted on $R$ or $H$, a contradiction.

Lemma 6. [6] Let $G$ be a $D V$ graph, minimally non $R D V, T$ a DV-model of $G$, $H$ a leaf of $T$ and $T[H, C]$ a branch of $T$. Then

1. Every edge of $T$ dominated by $H$ is in $T[H, C]$ or $T[\mathbf{D}]$.

2. If there is an edge of $T$ dominated by $H$ in $T(H, C]$ then there is one in $T[\mathbf{D}]$.

\subsection{Leafage 4}

In this section and in every section relative to leafage four, we assume the following notation. If $G$ is a DV graph and $T$ is a DV-model of $G$ with exactly four leaves, we call $H_{1}, H_{2}, H_{3}, H_{4}$ these leaves. If $|\mathbf{D}|=1$, then we call $C$ the vertex of degree four of $T$. If $|\mathbf{D}|=2$, then we call $C_{1}, C_{2}$ the vertices of degree three of $T$ and we assume that the four branches of $T$ are $T\left[H_{i}, C_{1}\right]$ for $i=1,2$ and $T\left[H_{j}, C_{2}\right]$ for $j=3,4$. 
Lemma 7. Let $G$ be a $D V$ non $R D V$ graph and $T$ a $D V$-model of $G$ with 4 leaves then:

1. If $|\mathbf{D}|=1$, then there is one vertex crossing $C$ in $T\left[H_{i}, H_{j}\right]$ and one vertex crossing $C$ in $T\left[H_{k}, H_{l}\right]$ being $\{i, j, k, l\}=\{1,2,3,4\}$.

2. If $|\mathbf{D}|=2$, then there is a vertex crossing $C_{1}$ in $T\left[H_{1}, H_{2}\right]$ if and only if there is a vertex crossing $\mathrm{C}_{2}$ in $\mathrm{T}\left[\mathrm{H}_{3}, \mathrm{H}_{4}\right]$.

Proof. 1. The tree $T$ cannot be rooted on $C$, so there is a vertex crossing $C$ in $T\left[H_{i}, H_{j}\right]$ for some $i \neq j \in\{1,2,3,4\}$. Suppose by symmetry that there is a vertex crossing $C$ in $T\left[H_{1}, H_{2}\right]$. If there is a vertex crossing $C$ in $T\left[H_{3}, H_{4}\right]$, then the lemma is true so we can assume that there is no vertex crossing $C$ in $T\left[H_{3}, H_{4}\right]$. The tree $T$ cannot be rooted on $H_{1}$ so there is a vertex crossing $C$ in $T\left[H_{2}, H_{i}\right]$ for some $i \in\{3,4\}$. By symmetry we can assume that there is a vertex crossing $C$ in $T\left[H_{2}, H_{3}\right]$. If there is a vertex crossing $C$ in $T\left[H_{1}, H_{4}\right]$, then the lemma is true so we can assume that there is no vertex crossing $C$ in $T\left[H_{1}, H_{4}\right]$. The tree $T$ cannot be rooted on $H_{2}$ so there is a vertex crossing $C$ in $T\left[H_{1}, H_{3}\right]$. But then $T$ is not a DV-model, a contradiction.

2. Suppose by contradiction that there is a vertex crossing $C_{1}$ in $T\left[H_{1}, H_{2}\right]$ and that there is no vertex crossing $C_{2}$ in $T\left[H_{3}, H_{4}\right]$. As $G$ is a DV graph, it is not possible that for each $i, j \in\{1,2\}$ there exists a vertex crossing $C_{1}$ in $T\left[H_{i}, C_{2}\right]$. Suppose by symmetry that there is no vertex crossing $C_{1}$ in $T\left[H_{1}, C_{2}\right]$. Then $T$ can be rooted on $\mathrm{H}_{2}$, a contradiction.

Theorem 3. Let $G$ be a $D V$ graph, minimally non $R D V$, with leafage four.

1. There are four cliques of $G$ that are always leaves in every $D V$-model.

2. Then $G$ has exactly one asteroidal quadruple.

Proof. 1. Let $T$ be a $D V$-model with four leaves $H_{1}, H_{2}, H_{3}, H_{4}$. Suppose that one of them is bad. In order to fix ideas let $H_{1}$ be bad. By Lemma 6 , there is $e=A B \in T[\mathbf{D}]$ dominated by $H_{1}$. Clearly $|\mathbf{D}|=2$. Let $C_{1}$ and $C_{2}$ be the vertex of $\mathbf{D}$ such that $H_{1}, H_{2} \in \operatorname{Bouq}\left(C_{1}\right)$ and $H_{2}, H_{4} \in \operatorname{Bouq}\left(C_{2}\right)$ and $B \in T\left[C_{1}, A\right]$. Let $T^{\prime}=T-e+A H_{1}$. If there is a vertex crossing $C_{1}$ in $T\left[H_{1}, H_{2}\right]$ then by Lemma 7 there is a vertex crossing $C_{2}$ in $T\left[H_{3}, H_{4}\right]$. Hence $T^{\prime}$ is a $R D V$-model rooted on $H_{3}$ if there is a vertex crossing $C_{2}$ in $T\left[H_{3}, C_{1}\right]$ or $H_{4}$ if there is a vertex crossing $C_{2}$ in $T\left[H_{4}, C_{1}\right]$.

If there is no vertex crossing $C_{1}$ in $T\left[H_{1}, H_{2}\right]$ then by Lemma 7 there is no vertex crossing $C_{2}$ in $T\left[H_{3}, H_{4}\right]$. Hence $T^{\prime}$ is a $R D V$-model rooted on $B$.

Therefore every $H_{i}$ is good so it is a leaf in every $D V$-model for $i=1,2,3,4$.

2. By Theorem 1 , there are cliques $H_{1}, H_{2}, H_{3}, H_{4}$ that are leaves in every $D V$-model of $G$. Let $T$ be a $D V$-model with four leaves, $C_{1}$ and $C_{2}$ the vertices of degree at least 3. Observe that $C_{1}$ may be $C_{2}$. Suppose $H_{1}, H_{2} \in \operatorname{Bouq}\left(C_{1}\right)$ and $H_{3}, H_{4} \in \operatorname{Bouq}\left(C_{2}\right)$. If there is another asteroidal quadruple there are four cliques $X_{1}, X_{2}, X_{3}, X_{4}$ such that $X_{1}, X_{2} \in \operatorname{Bouq}\left(C_{1}\right)$ and $X_{3}, X_{4} \in \operatorname{Bouq}\left(C_{2}\right)$ and at least one $X_{i} \neq H_{i}$ for some $i=1,2,3,4$. Let $T^{\prime}=T\left[X_{1}, X_{2}, X_{3}, X_{4}\right]$. Clearly $X_{i}$ is good in $T^{\prime}$ then $X_{i}$ are leaves in every clique tree of $G_{T^{\prime}}$. As at least one $X_{i} \neq H_{i}$ for some $i=1,2,3,4, G_{T^{\prime}}$ is a $R D V$ graph by minimality of $G$. Let $T^{\prime \prime}$ be a $R D V$-model of $G_{T^{\prime}}$ rooted on a vertex $R$. As every $X_{i}$ is a leaf of $T^{\prime \prime}$, thus it is possible to build a $R D V$-model of $G$ with $T\left[H_{i}, X_{i}\right]$ and $T^{\prime \prime}$ as follows: $T^{\prime \prime}+\sum_{i=1}^{4} T\left[H_{i}, X_{i}\right]$ rooted on $R$ or $H_{i}$ for some $i=1,2,3$, 4, a contradiction. 
Lemma 8. Let $G$ be a $D V$ non $R D V$ graph, $T$ a $D V$-model of $G$ with 4 leaves that does not have vertex crossing $C_{1}$ in $T\left[H_{1}, H_{2}\right]$ and $e \in T\left[C_{1}, C_{2}\right]$. Then there are two vertices $x, y \in l a b(e)$ such that $T_{x}$ and $T_{y}$ have different end towards $C_{1}$ and towards $C_{2}$.

Proof. Suppose by contradiction, that $e=A B$ with $B \in T\left[C_{1}, A\right]$ satisfies that for every vertex $x \in \operatorname{lab}(e)$ the end of $T_{x}$ toward $C_{1}$ is the same and suppose that it is in $T\left[B, H_{1}\right]$. Let $X$ be this end and $T^{\prime}=T-e+A X$. As there is no vertex crossing $C_{1}$ in $T\left[H_{1}, H_{2}\right]=$ $T^{\prime}\left[H_{1}, H_{2}\right]$, by Lemma 7 , there is no vertex crossing $C_{2}$ in $T\left[H_{3}, H_{4}\right]=T^{\prime}\left[H_{3}, H_{4}\right]$. On the other hand, the end of $T_{x}$ toward $H_{1}$ is $X$ then there is no vertex crossing $X$ in $T^{\prime}\left[H_{1}, A\right]$. Hence $T^{\prime}$ rooted on $B$ is a $R D V$-model of $G$, a contradiction.

Lemma 9. Let $G$ be a $D V$ non $R D V$ graph and $T$ a $D V$-model of $G$ with 4 leaves. There are not two edges with the same label one in a branch and the other in $T\left[C_{1}, C_{2}\right]$.

Proof. Suppose by contradiction, that $e$ and $e^{\prime}$ are two edges with the same label, one in $T\left[H_{1}, C_{1}\right]$ and the other in $T\left[C_{1}, C_{2}\right]$. Let $e=A B \in T\left[H_{1}, C_{1}\right]$ and $e^{\prime}=A^{\prime} B^{\prime} \in T\left[C_{1}, C_{2}\right]$ with $B \in T\left[A, C_{1}\right]$ and $B^{\prime} \in T\left[C_{1}, A^{\prime}\right]$. Let $T^{\prime}=T-\left\{e, e^{\prime}\right\}+A B^{\prime}+B A^{\prime}$. It is a $D V$-model of $G$.

If there is a vertex crossing $C_{1}$ in $T\left[H_{1}, H_{2}\right]$ then $B \neq C_{1}$ and as there is a vertex crossing $C_{1}$ in $T\left[H_{1}, C_{2}\right]$ then there is no vertex crossing $C_{1}$ in $T\left[H_{2}, C_{2}\right]$ because $G$ is a $D V$ graph. By Lemma 7 , there is a vertex crossing $C_{2}$ in $T\left[H_{3}, H_{4}\right]$. As $G$ is a $D V$-graph then there is no vertex crossing $C_{2}$ in $T\left[H_{3}, C_{1}\right]$ or there is no in $T\left[H_{4}, C_{1}\right]$. Suppose there is no vertex crossing $C_{2}$ in $T\left[H_{3}, C_{1}\right]$ then $T^{\prime}$ rooted on $H_{4}$ is a $R D V$-model of $G$, a contradiction.

If there is no vertex crossing $C_{1}$ in $T\left[H_{1}, H_{2}\right]$, by Lemma 7 there is no vertex crossing $C_{2}$ in $T\left[H_{3}, H_{4}\right]$. Hence $T^{\prime}$ rooted on $H_{1}$ is a $R D V$-model of $G$, a contradiction.

Lemma 10. Let $G$ be a $D V$, minimally non $R D V$ graph and $T$ a $D V$-model of $G$ with 4 leaves $T\left[H_{1}, C_{1}\right]$ is a branch with at least three vertices and e an edge of $T\left(H_{1}, C_{1}\right]$. If all the dominated edges of e are in $T\left[C_{1}, C_{2}\right]$ then the maximally farthest from e does not dominate edges of $T$.

Proof. Let $e^{\prime}=A^{\prime} B^{\prime}$ be the dominated edge of $e$ farthest away with $B^{\prime} \in T\left[C_{1}, A^{\prime}\right]$. By the choice of $e^{\prime}$, the subtree $T\left[A^{\prime}, H_{3}, H_{4}\right]$ of $T$ does not have edges dominated by $e^{\prime}$.

If $e^{\prime \prime}$ were an edge of $T$ dominated by $e^{\prime}$, i.e $l a b\left(e^{\prime \prime}\right) \subseteq l a b\left(e^{\prime}\right)$ then $e^{\prime \prime} \notin T\left[H_{2}, C_{1}\right]$ because of that $G$ is a $D V$ graph. Also $e^{\prime \prime} \notin T\left[B^{\prime}, H_{1}\right)$ because in this case lab $\left(e^{\prime}\right)=\operatorname{lab}\left(e^{\prime \prime}\right)$ contradiction by Lemmas 4 or 9 . Then $e^{\prime \prime}=H_{1} A_{1}$. Let $T^{\prime}=T-\left\{e^{\prime}, e^{\prime \prime}\right\}+A^{\prime} A_{1}+B^{\prime} H_{1}$. If there is no vertex crossing $C_{1}$ in $T\left[H_{1}, H_{2}\right]$ then $T^{\prime}$ rooted on $H_{1}$ is a $R D V$-model of $G$, a contradiction. If there is a vertex crossing $C_{1}$ in $T\left[H_{1}, H_{2}\right]$ then $T^{\prime}$ rooted on $H_{3}$ or $H_{4}$, depending on which vertex crossing $C_{2}$ is absent, is a $R D V$-model of $G$, a contradiction.

Lemma 11. Let $G$ be a $D V$, minimally non $R D V$ graph and $T$ a $D V$-model of $G$ with four leaves such that has a vertex crossing $C_{1}$ in $T\left[H_{1}, H_{2}\right]$ and $H_{2} C_{1}, H_{4} C_{2} \in E(T)$. Let $e \in T\left[C_{1}, C_{2}\right]$ with $|l a b(e)|$ minimum then e does not dominate simultaneously two edges one in $T\left[H_{1}, C_{1}\right]$ and the other in $T\left[H_{3}, C_{2}\right]$.

Proof. Suppose by contradiction that $e$ dominates simultaneously an edge $e_{0} \in T\left[H_{1}, C_{1}\right]$ and $e_{0}^{\prime} \in T\left[H_{3}, C_{2}\right]$. Among all the edges in these conditions choice $e_{0}$ and $e_{0}^{\prime}$ at minimum distance. Let $e_{0}=A_{0} B_{0}$ and $e_{0}^{\prime}=A_{0}^{\prime} B_{0}^{\prime}$ with $B_{0}, B_{0}^{\prime} \in T\left[A_{0}, A_{0}^{\prime}\right]$. Observe that $B_{0}^{\prime} \neq C_{2}$ and $B_{0} \neq C_{1}$ by the existence of vertices crossing $T\left[H_{i}, H_{i+1}\right]$ for $i=1,3$. By the election 
of $e_{0}^{\prime}, B_{0}$ does not dominate an edge in $T\left[B_{0}^{\prime}, C_{2}\right]$. If $B_{0}$ dominates an edge $e^{\prime}$ in $T\left[C_{1}, e\right]$ with $e^{\prime}=A B$ and $B \in T\left[B_{0}, A\right]$ then we build $T^{\prime}=T-\left\{e^{\prime}, e_{0}\right\}+A B_{0}+A_{0} B$ is a $R D V$ model of $G$ rooted on $H_{3}$. If $B_{0}$ dominates edges an edge $e^{\prime}$ in $T\left(e, C_{2}\right]$ then $l a b\left(e^{\prime}\right) \subseteq l a b(e)$ and by Lemma $4, l a b\left(e^{\prime}\right) \neq l a b(e)$. So $\left|l a b\left(e^{\prime}\right)\right|<|l a b(e)|$ a contradiction. If $B_{0}$ dominates $H_{2} C_{1}$ then as $e$ dominates $e_{0}$ and $G$ is a $D V$-model every vertex of $l a b\left(H_{2} C_{1}\right)$ are twins in $G$ contradicting Theorem 2. Therefore $B_{0}$ does not dominate edges in $T\left[H_{2}, B_{0}^{\prime}, H_{4}\right]$. Analogously $B_{0}^{\prime}$ does not dominate edges in $T\left[H_{2}, B_{0}, H_{4}\right]$. As $H_{i}$ does not dominate edges in $T\left[B_{0}, B_{0}^{\prime}, H_{j}\right]$ for $i \neq j, i, j \in\{2,4\}$ then there are an asteroidal quadruple whose vertices are in $B_{0}, B_{0}^{\prime}, H_{2}, H_{4}$ contradicting Theorem 2.

Lemma 12. Let $G$ be a $D V$ non $R D V$ graph and $T$ a $D V$-model of $G$ with 4 leaves. If $T$ does not have vertex crossing $C_{1}$ in $T\left[H_{1}, H_{2}\right]$ then for every vertex of $T\left[C_{1}, C_{2}\right]$ has a vertex crossing.

Proof. Suppose by contradiction, that there is a vertex $X$ without vertex crossing $X$ in $T\left[C_{1}, C_{2}\right]$. Then $T$ rooted on $X$ is a $R D V$-model of $G$, a contradiction.

\section{DV minimally non RDV graphs and some special connections.}

Special connection linking vertices $a_{1}$ and $a_{2}$ in a graph were defined in [2] in order to characterize directed path graph by forbidden asteroidals. Here, we will need only two types of these connections:

- Type 1: vertices $a_{1}, a_{2}, w$ and edges $a_{1} w, a_{2} w$.

- Type 2: vertices $a_{1}, a_{2}, a, b, c, d$ and edges $a_{1} a, a_{1} b, a_{2} c, a_{2} d, a b, b c, c d, d a, a c$.

Lemma 13. [1] Let $G$ be a directed path and let $a_{1}$ and $a_{2}$ two non adjacent vertices that are linked by a special connection of Type 1 or Type 2. Then for every DV-model $T$ of $G$, the subpath $T\left(a_{1}, a_{2}\right)$ is a directed path.

As was proved in [6], this kind of connections are not the only that appears in DV minimally non RDV graphs.

In this section, like in the previous one, we will study special connections of Type 1 or Type 2 according to the leafage of the graph.

\subsection{Any leafage}

Theorem 4. Let $G$ be a $D V$, minimally non $R D V$ graph, $T$ a $D V$-model of $G$ with minimum number of leaves and then maximizing $T[\mathbf{D}]$, e and $e^{\prime}$ edges in two branches of $T$ with the same label. Then the simplicial vertices of the leaves of $T$ in those branches are linked by a special connection of Type 1 or Type 2.

Proof. Let $T\left[H_{1}, C\right]$ and $T\left[H_{2}, C^{\prime}\right]$ be the branches that contain $e$ and $e^{\prime}$ and $a_{1}, a_{2}$ simplicial vertices of $H_{1}$ and $H_{2}$ respectively. By Theorem 2, $G$ does not have false twins then $e$ and $e^{\prime}$ are not incident in $H_{1}$ and $H_{2}$ (otherwise $a_{1}$ and $a_{2}$ are false twins). If one of them is incident in a leaf, for example $e$ in $H_{1}$ then every vertex of $l a b\left(e^{\prime}\right)$ has the same end equal $H_{1}$ in the direction of $C$ contradicting Lemma 3. Hence none of them is incident in a leaf.

Let $e \in T\left[H_{1}, C\right]$ and $e^{\prime} \in T\left[H_{2}, C^{\prime}\right]$ maximizing the distance among all pair of edges with the same label. 
Next, we will prove that $H_{i}$ is good for $i=1,2$. Suppose by contradiction, that $H_{1}$ is bad. Then by Lemma 6 , it has a dominated edge $e_{0}$ in $T[\mathbf{D}]$ and no one in other branch different from $T\left[H_{1}, C\right]$. As $l a b(e)=l a b\left(e^{\prime}\right)$ and $T$ is a $D V$-model then $e_{0} \in T\left[C, C^{\prime}\right]$. Then $e, e_{0}, e^{\prime}$ appear in this order along $T\left[H_{1}, H_{2}\right]$ and as $l a b(e)=l a b\left(e^{\prime}\right)$ then $l a b\left(e^{\prime}\right) \subset$ $l a b\left(e_{0}\right) \subset H_{1}$ i.e $H_{1}$ dominates $e^{\prime} \in T\left[H_{2}, C^{\prime}\right]$ which contradicts the Lemma 6 . Therefore, $H_{1}$ is good. Analogously, $H_{2}$ is good.

As $\left|E\left(T\left[H_{1}, C\right]\right)\right|>1$ we have $H_{1}$ has a neighbor $A_{1}$ with $A_{1} \neq C$. Analogously if $A_{2}$ is the neighbor of $H_{2}$ then $A_{2} \neq C^{\prime}$. Let $e_{1}=A_{1} B_{1} \in T\left[H_{1}, C\right], B_{1}$ may be $C$. Analogously, let $e_{2}=A_{2} B_{2} \in T\left[H_{2}, C^{\prime}\right]$. By Lemma 5 , there exists dominated edges of $e_{1}$ and $e_{2}$ respectively. Let $e_{1}^{\prime}$ and $e_{2}^{\prime}$ be maximally farthest from $e_{1}$ and $e_{2}$ respectively (i.e there is no edge $e_{i}^{\prime \prime}$ dominated by $e_{i}$ and distinct from $e_{i}^{\prime}$ such that $e_{i}^{\prime}$ is between $H_{i}$ and $e_{i}^{\prime \prime}$ for $i=1,2)$. As $l a b(e)=l a b\left(e^{\prime}\right)$ and $T$ is a $D V$-model then $e_{1}^{\prime}, e_{2}^{\prime} \in T\left[H_{1}, H_{2}\right]$.

Observe that if $e_{1}^{\prime} \in T[\mathbf{D}]$ as $\operatorname{lab}\left(e_{1}^{\prime}\right) \subseteq \operatorname{lab}\left(e_{1}\right)$ then $\operatorname{lab}\left(e_{1}^{\prime}\right) \subseteq \operatorname{lab}(e)=\operatorname{lab}\left(e^{\prime}\right)$. As $e^{\prime} \in T\left[H_{2}, C^{\prime}\right], \operatorname{lab}\left(e^{\prime}\right) \subset \operatorname{lab}(e)$ and $e_{1}^{\prime} \in T\left[C, C^{\prime}\right]$ then $\operatorname{lab}\left(e^{\prime}\right) \subseteq \operatorname{lab}\left(e_{1}^{\prime}\right)$. Therefore $l a b\left(e_{1}^{\prime}\right)=\operatorname{lab}\left(e^{\prime}\right)$ so $e^{\prime}$ is dominated by $e_{1}$ which contradicts the choice of $e_{1}^{\prime}$. Therefore $e_{1}^{\prime} \notin T[\mathbf{D}]$. Analogously $e_{2}^{\prime} \notin T[\mathbf{D}]$.

In case that $e_{1}^{\prime}$ is incident in $H_{2}$, by Lemma 3 there is $x \in \operatorname{lab}\left(e_{1}^{\prime}\right)$ such that $x \in H_{1} \cap H_{2}$. Therefore, $a_{1}$ and $a_{2}$ are linked by a special connection of Type 1 . Analogously if $e_{2}^{\prime}$ is incident in $H_{1}$.

Finally, suppose $e_{1}^{\prime}$ and $e_{2}^{\prime}$ are not incident in $H_{1}$ and $H_{2}$ respectively. As $e_{1}^{\prime} \in T\left[A_{2}, C^{\prime}\right]$, $e_{2}^{\prime} \in T\left[A_{1}, C\right]$ and $\operatorname{lab}\left(e_{2}^{\prime}\right) \subset A_{2}$ we have $l a b\left(e_{2}^{\prime}\right) \subset \operatorname{lab}\left(e_{1}^{\prime}\right)$. Similarity, $\operatorname{lab}\left(e_{1}^{\prime}\right) \subset \operatorname{lab}\left(e_{2}^{\prime}\right)$. Hence $l a b\left(e_{1}^{\prime}\right)=\operatorname{lab}\left(e_{2}^{\prime}\right)$. By the choice of $e$ and $e^{\prime}$, if $e_{2}^{\prime}$ is between $e$ and $H_{1}$ then $e_{1}^{\prime}$ is between $e^{\prime}$ and $C^{\prime}$. In this case $l a b(e) \subseteq \operatorname{lab}\left(e_{1}^{\prime}\right)=\operatorname{lab}\left(e_{2}^{\prime}\right)$ and $l a b\left(e_{1}^{\prime}\right)=\operatorname{lab}\left(e_{2}^{\prime}\right) \subseteq l a b(e)$ then $\operatorname{lab}\left(e_{1}^{\prime}\right)=l a b(e)$ then $l a b\left(e_{1}^{\prime}\right)=l a b(e)=\operatorname{lab}\left(e^{\prime}\right)=l a b\left(e_{2}^{\prime}\right)$. Hence $e_{1}^{\prime}=e^{\prime}$ and $e_{2}^{\prime}=e$ because in other case Lemma 4 is contradicted. Therefore, every vertex in $l a b\left(e_{1}^{\prime}\right)=\operatorname{lab}\left(e_{2}^{\prime}\right)$ is in $A_{1} \cap A_{2}$ and exist $x, y \in A_{1} \cap A_{2}$ being $x \in H_{1}$ and $y \in H_{2}$. Among all $x \in H_{1} \cap A_{2}$ we chose one that maximizes $\left|C_{x}\right|$. Analogously for $y$. If $x \in H_{2}$ or $y \in H_{1}$ then $a_{1}$ and $a_{2}$ are linked by a special connection of Type 1 .

Suppose $x \in H_{1} \backslash H_{2}$ and $y \in H_{2} \backslash H_{1}$. By Lemma 3, there are $x_{1} \neq x$ and $x_{2} \neq y$ with $x_{1} \in H_{1} \cap A_{1}$ and $x_{2} \in H_{2} \cap A_{2}$ respectively. Among all the $x_{1} \in H_{1} \cap A_{1}$ we chose one minimizing $\left|C_{x_{1}}\right|$, analogously for $x_{2}$. If $x_{1}$ and $x_{2}$ were adjacent some of them would be in $l a b\left(e_{1}^{\prime}\right)$. Suppose $x_{1} \in \operatorname{lab}\left(e_{1}^{\prime}\right)$ then $x_{1} \in \operatorname{lab}\left(e_{2}\right)$ and $x_{1} \in H_{1} \cap A_{2}$. As $x_{1}$ was chosen minimizing $\left|C_{x_{1}}\right|$ then $C_{x}=C_{x_{1}}$ and $x$ and $x_{1}$ are twins in $G$, a contradiction. Therefore $x_{1}$ and $x_{2}$ are not adjacent. So $a_{1}$ and $a_{2}$ are linked by a special connection of Type 2 .

In the following we need to introduce some notation because some edges of a $D V$-model play an important role.

From now until the end we use the following notation.

Let $T\left[H_{i}, C\right]$ be a branch of $T$ with $\left|V\left(T\left[H_{i}, C\right]\right)\right|>2$. We denote $e_{i}=A_{i} B_{i}$, the edge of $T\left[H_{i}, C\right]$ such that $A_{i}$ is the neighbor of $H_{i}$ and $B_{i} \neq H_{i}$. Since $e_{i} \in T\left(H_{i}, C\right]$ then by Lemma 5 there is at least an edge of $T$ dominated edge by $e_{i}$. Choose $e_{i}^{\prime}$ a maximally farthest from $e_{i}$ (i.e there is no edge $e_{i}^{\prime \prime}$ dominated by $e_{i}$ and distinct from $e_{i}^{\prime}$ such that $e_{i}^{\prime}$ is between $H_{i}$ and $e_{i}^{\prime \prime}$. We denote $e_{i}^{\prime}=A_{i}^{\prime} B_{i}^{\prime}$ with $B_{i}^{\prime} \in T\left[A_{i}^{\prime}, C\right]$. By Lemma $6, e_{i}^{\prime}$ is not in the same branch of $e_{i}$.

Corollary 2. Let $G$ be a $D V$, minimally non $R D V$ graph, $T$ a $D V$-model of $G$ with minimum number of leaves and then maximizing $T[\mathbf{D}], H_{1}, H_{2}$ leaves of $T, T\left[H_{1}, C\right], T\left[H_{2}, C^{\prime}\right]$ 
branches of $T, e_{1}^{\prime} \in T\left[H_{2}, C^{\prime}\right]$ and $e_{2}^{\prime} \in T\left[H_{1}, C\right]$. Then the simplicial vertices of $H_{1}$ and $\mathrm{H}_{2}$ are linked by a special connection of Type 1 or Type 2.

Proof. If $e_{1}^{\prime}=H_{2} A_{2}$ or $e_{2}^{\prime}=H_{1} A_{1}$, by Lemma 3 there is $x \in H_{1} \cap H_{2}$. Hence the simplicial vertices of $H_{1}$ and $H_{2}$ are linked by a special connection of Type 1. Otherwise, as $e_{1}^{\prime} \in T\left[H_{2}, C^{\prime}\right]$ and $e_{2}^{\prime} \in T\left[H_{1}, C\right]$ then $\operatorname{lab}\left(e_{1}^{\prime}\right)=\operatorname{lab}\left(e_{2}^{\prime}\right)$. So by Theorem 4 , the simplicial vertices of $H_{1}$ and $H_{2}$ are linked by a special connection of Type 1 or Type 2.

\subsection{Leafage 4}

We assume the same notation of subsections 4.2 and 5.1.

Lemma 14. Let $G$ be a $D V$ non $R D V$ graph and $T$ a $D V$-model of $G$ with 4 leaves then maximizing $T[\mathbf{D}]$. If $e_{1}^{\prime} \in T\left[H_{i}, C_{j}\right]$ with $i \neq 1$ then the simplicial vertices of $H_{1}$ and $H_{i}$ are linked by a special connection of Type 1 or Type 2.

Proof. In case $e_{1}^{\prime}=H_{i} A_{i}$, observe that $A_{i}$ may be $C_{j}$, since $l a b\left(e_{1}^{\prime}\right) \subseteq \operatorname{lab}\left(e_{1}\right)$ and by Lemma 3, there is a vertex $x$ in $l a b\left(e_{1}^{\prime}\right)$ such that $x \in H_{1}$ then the simplicial vertices of $H_{1}$ and $H_{2}$ are linked by a special connection of Type 1.

Suppose $e_{1}^{\prime} \neq H_{i} A_{i}$. By Lemma 5 , we know that $e_{i}^{\prime} \notin T\left[H_{i}, C_{j}\right]$. As $T$ is a $D V$ model and $e_{1}^{\prime} \in T\left[H_{i}, C_{j}\right]$ then $e_{i}^{\prime} \in T\left[H_{1}, C_{2}\right]$. By Lemma 9, $e_{i}^{\prime} \notin T\left[C_{1}, C_{2}\right]$ then $e_{i}^{\prime} \in$ $T\left[H_{1}, C_{1}\right]$. Hence, by Corollary 2 , the simplicial vertices of $H_{1}$ and $H_{2}$ are linked by a special connection of Type 1 or Type 2 .

\section{DV non RDV graphs with leafage 4 .}

In this section we propose formally our conjecture and proved it for graphs with leafage four.

As was mentioned before, special connections of Type 1 and Type 2 force the direction in any DV-model of the graph but we know that this kind of connections are not the only that produce this effect in the model. Although we could not describe every those special connections we can give a conjecture using a more abstract concept: strong path.

Two non adjacent vertices $a_{i}, a_{j}$ of a DV graph $G$ are linked by a strong path if in every DV-model $T$ of $G, T\left(a_{i}, a_{j}\right)$ is a directed path.

Conjecture: A graph is DV non RDV graph if and only if it has an asteroidal quadruple partitioned in two pairs each one linked by a strong path.

As we said before if a graph is $D V$ non $R D V$ it has an asteroidal quadruple hence its leafage is at least four. In case the graph is minimal in the difference of both classes we have proved that it has only one asteroidal quadruple.

In this section we will prove the conjecture in graph of leafage four, before we will prove some previous results. We assume the same notation of subsections 4.2 and 5.1.

Property 1. Let G be a DV graph and it does not have an asteroidal quadruple. If there is T a DV model with four leaves satisfying:

1. $\left|V\left(T\left[H_{2}, C_{1}\right]\right)\right|=\left|V\left(T\left[H_{4}, C_{2}\right]\right)\right|=2$,

2. $H_{1}, H_{2}, H_{4}$ are good, 
3. there are $x_{i}, y_{i} \in H_{i}$ for $i=2,4$ with $\left|C_{x_{i}}\right|>\left|C_{y_{i}}\right|>1$ and $x_{2} \operatorname{crossing} C_{1}$ in $T\left[H_{1}, H_{2}\right]$ respectively $x_{4}$ crossing $C_{2}$ in $T\left[H_{3}, H_{4}\right]$.

then there are a succession of edges $e_{1}, . ., e_{k}$ in $T\left[H_{1}, H_{3}\right]$ with $l a b\left(e_{1}\right) \subseteq H_{3}, l a b\left(e_{i}\right) \subseteq$ $l a b\left(e_{i+1}\right), e_{k} \in T\left[C_{1}, C_{2}\right]$ and if $k>1 e_{2 i+1} \in T\left[H_{1}, C_{1}\right]$ and $e_{2 i} \in T\left[C_{2}, H_{3}\right]$ whether $k \neq 2 i, 2 i+1$

Proof. Let $T_{x_{i}}=T\left[L_{i}, H_{i}\right], i=2,4$. Due to $x_{2}$ is a vertex crossing $C_{1}$ in $T\left[H_{1}, H_{2}\right]$, $L_{2} \neq C_{1}$; analogously $L_{4} \neq C_{2}$. As $G$ does not have an asteroidal quadruple then $H_{3}$ is a bad leaf in $T\left[H_{1}, H_{2}, H_{4}\right]$. So there is an edge in $T\left[H_{1}, H_{2}, H_{4}\right]$ dominated by $H_{3}$. By the existence of $y_{i}$ such edge is not $H_{2} C_{1}$ or $H_{4} C_{2}$. Let $e_{1}$ be the nearest $H_{3}$. If $e_{1} \in T\left[C_{1}, C_{2}\right]$, we are done. If $e_{1} \notin T\left[C_{1}, C_{2}\right]$ then $e_{1} \in T\left[H_{1}, C_{1}\right]$. As $G$ is a $D V$ graph $e_{1} \in T\left[H_{1}, L_{2}\right]$. Let $e_{1}=X_{1} Y_{1}$ with $X_{1} \in T\left[Y_{1}, C_{1}\right]$. By the choice of $e_{1}$, it is clear that $H_{3}$ is good in $T\left[H_{2}, H_{4}, X_{1}\right]$. As $G$ does not have an asteroidal quadruple, $X_{1}$ must be bad in $T\left[H_{2}, H_{3}, H_{4}\right]$. As before exposed, there is an edge there dominated by $X_{1}$ which is not $H_{2} C_{1}$ or $H_{4} C_{2}$ because there exists $y_{i}$ for $i=2$, 4 . So it is in $T\left[C_{1}, H_{3}\right]$. Let $e_{2}$ the nearest $X_{1}$. Clearly $e_{1}, e_{2}, H_{3}$ appear in this order in $T\left[H_{1}, H_{3}\right]$ and as $l a b\left(e_{1}\right) \subseteq H_{3}$ then $l a b\left(e_{1}\right) \subseteq l a b\left(e_{2}\right)$. If $e_{2} \in T\left[C_{1}, C_{2}\right]$, we are done. If $e_{2} \in T\left[C_{2}, H_{3}\right]$ as $G$ is a $D V$ graph then $e_{2} \in T\left[L_{4}, H_{3}\right]$. Let $e_{2}=X_{2} Y_{2}$ with $X_{2} \in T\left[L_{2}, Y_{2}\right]$. Recursively applying the above reasoning the theorem is proved.

Property 2. Let G be a DV graph and it does not have an asteroidal quadruple. If there is $\mathrm{T}$ a DV model with four leaves satisfying:

1. $\left|V\left(T\left[H_{2}, C_{1}\right]\right)\right|=\left|V\left(T\left[H_{4}, C_{2}\right]\right)\right|=2$,

2. $H_{1}, H_{2}, H_{4}$ are good,

3. there are $x_{i}, y_{i} \in H_{i}$ for $i=2,4$ with $\left|C_{x_{i}}\right|>\left|C_{y_{i}}\right|>1$ and $x_{2} \operatorname{crossing} C_{1}$ in $T\left[H_{1}, H_{2}\right]$ respectively $x_{4}$ crossing $C_{2}$ in $T\left[H_{3}, H_{4}\right]$.

4. there are not vertices crossing by $C_{1}$ nor $C_{2}$ in $T\left[H_{2}, H_{4}\right]$

then there is a RDV-model of $G$ rooted on $H_{4}$.

Proof. By Property 1 , there is a succession of edges $e_{1}, . ., e_{k}$. Let

$$
e_{i}=\left\{\begin{array}{l}
O E, \text { if } i=k \\
X_{i} Y_{i}, \text { with } X_{i} \in T\left[Y_{i}, C_{1}\right] \text { if } i \text { is odd } \\
X_{i} Y_{i}, \text { with } X_{i} \in T\left[Y_{i}, C_{2}\right] \text { if } i \text { is even }
\end{array}\right.
$$

Let $T_{1}=T-e_{1}+Y_{1} H_{3}$ if $k>1$ let

$$
T_{i}=\left\{\begin{array}{l}
T_{i-1}-e_{i}+Y_{i} X_{i-1}, \text { if } i<k \\
T_{i-1}-e_{i}+O X_{i-1}, \quad \text { if } i=k \text { and } k \text { is odd } \\
T_{i-1}-e_{i}+E X_{i-1}, \quad \text { if } i=k \text { and } k \text { is even }
\end{array}\right.
$$

Clearly each $T_{i}$ for $1 \leq i \leq k$ is a $D V$-model of $G$ and $T_{k}$ is a $R D V$-model of $G$ rooted on $\mathrm{H}_{4}$.

Property 3. Let $G$ be a DV minimally non RDV graph, $T$ a DV-model of $G$ with four leaves satisfying:

1. maximizing $T[\mathbf{D}]$,

2. $\left|V\left(T\left[H_{2}, C_{1}\right]\right)\right|=\left|V\left(T\left[H_{4}, C_{2}\right]\right)\right|=2$, 
3. there is no vertex crossing $C_{1}$ in $T\left[H_{1}, H_{2}\right]$

then every $T^{\prime}$, DV-model of $G$, has four leaves.

Proof. By Lemma 3 there are at least two non simplicial vertices in $H_{i}$ for $i=1,2,3,4$. Let $x_{i}, y_{i}$ be non simplicial vertices of $H_{i}$ being $\left|C_{x_{i}}\right|$ maximum for $i=1,2,3,4$.

Claim If there is $e_{1} \in T\left(H_{1}, C_{1}\right]$ then $C_{x_{2}} \backslash H_{2} \subset C_{x_{1}}$ or $C_{x_{2}} \backslash\left\{H_{2}, H_{4}\right\} \subset C_{x_{1}}$.

Proof. By Lemma 5, there is a dominated edge of $e_{1}$ outside $T\left(H_{1}, C_{1}\right]$. As $T$ does not have vertex crossing $C_{1}$ in $T\left[H_{1}, H_{2}\right]$ then $e_{1}^{\prime} \neq C_{1} H_{2}$. Hence $e_{1}^{\prime} \in T\left[C_{1}, H_{3}, H_{4}\right]$. In case $e_{1}^{\prime} \in T\left[C_{1}, C_{2}\right]$ as $l a b\left(e_{1}^{\prime}\right) \subset l a b\left(e_{1}\right) \subset A_{1}$ by Lemma 8 there is a vertex $x_{1} \in \operatorname{lab}\left(e_{1}^{\prime}\right) \cap H_{1}$ so we are done. Analogously if $e_{1}^{\prime} \in T\left[H_{3}, H_{4}\right]$.

Now we will prove that any $D V$-model has four leaves. If $\left|V\left(T\left[H_{1}, C_{1}\right]\right)\right|=2$ we can rename the leaves $H_{1}, H_{2}$ such that $C_{x_{2}} \backslash H_{2} \subset C_{x_{1}}$ or $C_{x_{2}} \backslash\left\{H_{2}, H_{4}\right\} \subset C_{x_{1}}$. Hence suppose without loss of generality that $C_{x_{2}} \backslash H_{2} \subset C_{x_{1}}$ or $C_{x_{2}} \backslash\left\{H_{2}, H_{4}\right\} \subset C_{x_{1}}$ and $C_{x_{4}} \backslash H_{4} \subset C_{x_{3}}$ or $C_{x_{4}} \backslash\left\{H_{2}, H_{4}\right\} \subset C_{x_{3}}$.

Let $T^{\prime}$ be a $D V$-model of $G$. As $G$ is $D V$ non $R D V, G$ has an asteroidal quadruple then $\ln \left(T^{\prime}\right) \geq 4$. By Theorem $1, H_{i}$ are leaves of $T^{\prime}$ for $i=1,2,3,4$. Let $X$ and $Y$ be vertices of $T^{\prime}$ such that $T^{\prime}\left[H_{1}, H_{3}\right] \cap T^{\prime}\left[H_{4}, X\right]=\{X\}$ and $T^{\prime}\left[H_{1}, H_{3}\right] \cap T^{\prime}\left[H_{2}, Y\right]=\{Y\}$. By the existence of $x_{i}$ for $i=1,2,3,4$ it is clear that $X H_{4}$ and $Y H_{2}$ are edges of $T^{\prime}$. Suppose $\ln \left(T^{\prime}\right)>4$, let $H$ be a leaf of $T^{\prime}$ different from $H_{i}$ for $i=1,2,3,4$. If $H$ dominates $Y H_{2}$ as $\left|l a b\left(Y H_{2}\right)\right|>1$ then $x_{2}, y_{2}$ are twins of $G$ contradicting Theorem 2. Hence $H$ does not dominate $Y H_{2}$, analogously $H$ does not dominate $X_{4}$. Therefore, $H$ must have a dominated edge in $T^{\prime}\left[H_{1}, H_{3}\right]$, otherwise $H_{1}, H_{2}, H_{3}, H$ or $H_{1}, H_{3}, H_{4}, H$ are good leaves contradicting that $G$ has one and only one asteroidal quadruple. Let $e$ be a dominated edge by $H$ in $T^{\prime}\left[H_{1}, H_{3}\right]$ maximally farthest from $H$ and $T_{1}^{\prime}, T_{2}^{\prime}$ the connected component of $T^{\prime}-e$ being $H \in T_{1}^{\prime}$. Note that vertices of $l a b(e)$ are twins in $G_{T_{1}^{\prime}}$.

By the choice of $e$ and as $T^{\prime}$ is a $D V$-model, every $e^{\prime}$ dominated edge by $e$ if some exists, is between $e$ and $H$ and then $l a b(e)=l a b\left(e^{\prime}\right)$.

Hence, in case $e^{\prime}$ exists, $T^{\prime}$ has two edges with the same label. As a consequence of Lemma 4 and Lemma 9 in $T$ equivalents edges to $e$ and $e^{\prime}$ respectively are in different branches. As $\left|V\left(T\left[H_{2}, C_{1}\right]\right)\right|=\left|V\left(T\left[H_{4}, C_{2}\right]\right)\right|=2, H_{2}, H_{4}$ are good and by Lemma 3 then none of them is in $T\left[H_{2}, C_{1}\right]+T\left[H_{4}, C_{2}\right]$ then they are one in $T\left(H_{1}, C_{1}\right]$ and the other $T\left(H_{3}, C_{2}\right]$. By Theorem 4 , the simplicial vertices of $H_{1}$ and $H_{3}$ are linked by a special connection of Type 1 or Type 2. If the connection of Type 1 then there is $x$ a vertex of $G$ such that $V(T) \backslash\left\{H_{2}, H_{4}\right\} \subset C_{x}$. By the existence of $x, x_{2}, x_{4}$ it is clear $\ln \left(T^{\prime}\right)=4$. If the connection of Type 2 then there are vertices $x, y, x_{0}, y_{0}$ satisfying $V(T) \backslash\left\{H_{2}, H_{3}, H_{4}\right\} \subset C_{x}, V(T) \backslash\left\{H_{1}, H_{2}, H_{4}\right\} \subset C_{y}, x_{0} \in H_{1}, y_{0} \in H_{3}$ with $C_{x_{0}} \cap C_{y_{0}}=\emptyset$. By the existence of $x, y, x_{0}, y_{0}, x_{2}, x_{4}$, it is easy to see that $\ln \left(T^{\prime}\right)=4$, a contradiction.

In case $e$ does not have dominated edges then it is the only edge whose label is equal lab(e). Let $\widetilde{e}$ be the equivalent edge of $e$ in $T$ and $T_{1}, T_{2}$ the connected component of $T-e$. Clearly $V\left(T_{i}^{\prime}\right)=V\left(T_{i}\right)$ for $i=1,2$ and vertices of $l a b(\widetilde{e})=l a b(e)$ are twins in $G_{T_{1}}$. As $e$ does not have dominated edges and does not dominate $H_{i}$ for $i=1,2,3,4$ then $\widetilde{e}$ must be in $T\left[C_{1}, C_{2}\right]$. If $H_{1} \in V\left(T_{1}\right)\left(H_{3} \in V\left(T_{1}\right)\right)$ then vertices of lab( $\left.\widetilde{e}\right)$ have the same extreme to $C_{1}\left(C_{2}\right)$ contradicting Lemma 8 .

We will say that $a_{1}$ and $a_{2}$ are linked by a strong connection if for every DV-model $T$ of $G$, the subpath $T\left(a_{1}, a_{2}\right)$ is a directed path. 
Theorem 5. A graph with leafage four is a DV non RDV graph if and only if it has an asteroidal quadruple $a_{1}, . ., a_{4}$ such that $a_{i}, a_{j}$ and $a_{k}, a_{l}$ are linked by a strong connection being $\{i, j, l, k\}=\{1,2,3,4\}$.

Proof. $\Leftarrow$ Trivial.

$\Rightarrow$ Let $G$ be a $D V$ minimally non $R D V$ graph. By Corollary $1, l(G)>3$ then as the hold graphs has four leaves so $l(G)=4$. Let $T$ be a $D V$-model with $\ln (T)=4$ and then maximizing $T[\mathbf{D}]$. By Theorem 1 we know that $G$ has an asteroidal quadruple. As $G$ is $D V$ minimally non $R D V$ the simplicial vertices of the leaves $H_{1}, H_{2}, H_{3}, H_{4}$ of $T$ are an asteroidal quadruple in $G$. We denote them by $a_{1}, a_{2}, a_{3}, a_{4}$ respectively.

In case $T$ is a star we will analyze the following situations:

- $\mid V\left(T\left[H_{i}, C\right]\right)=2$ for all $i \in\{1,2,3,4\}$ then by Lemma 7 , we are done.

- $\left|V\left(T\left[H_{i}, C\right]\right)\right|>2$ for some $i \in\{1,2,3,4\}$, to fix ideas $\left|V\left(T\left[H_{1}, C\right]\right)\right|>2$, then by Lemma 5 there is $e_{1}^{\prime}$ such that $e_{1}^{\prime} \in T\left[H_{j}, C\right]$ for $j \neq 1$, to fix ideas $j=2$. Therefore there is a special connection of Type 1 or 2 between $a_{1}$ and $a_{2}$.

- $\left|V\left(T\left[H_{j}, C\right]\right)\right|>2$ for $j \in\{3,4\}$ then there is $e_{j}^{\prime}$. By the type of connection between $a_{1}$ and $a_{2}, e_{j}^{\prime} \notin T\left[H_{1}, H_{2}\right]$ then there is a special connection between $a_{3}$ and $a_{4}$ of Type 1 or 2 , we are done.

- $\left|V\left(T\left[H_{j}, C\right]\right)\right|=2$ for $j=3,4$. Clearly if there is a vertex crossing $C$ in $T\left[H_{3}, H_{4}\right]$ then there is a special connection between $a_{3}$ and $a_{4}$ of Type 1 or 2 , we are done. Suppose there is no vertex crossing $C$ in $T\left[H_{3}, H_{4}\right]$. Then by Lemma 2 , there are vertices crossing $C$ in $T\left[H_{3}, H_{1}\right]$ and $T\left[H_{4}, H_{2}\right]$ or viceversa. Suppose that there are vertices crossing $C$ in $T\left[H_{3}, H_{1}\right]$ and $T\left[H_{4}, H_{2}\right]$.

On the other hand, there are four vertices $v_{1}, v_{2} \in H_{4} \cap C$ and $v_{1}^{\prime}, v_{2}^{\prime} \in H_{3} \cap C$ such that $C_{v_{i}} \cap C_{v_{j}^{\prime}}=\{C\}$. Suppose that $\left|C_{v_{2}}\right|$ and $\left|C_{v_{2}^{\prime}}\right|$ are maximum. If $v_{2} \in H_{1}$ and $v_{2}^{\prime} \in H_{2}$, there is a special connection between $a_{1}$ and $a_{4}$ of Type 1 or 2 and a special connection between $a_{2}$ and $a_{3}$ of Type 1 or 2 , we are done. Analogously if $v_{2} \in H_{2}$ and $v_{2}^{\prime} \in H_{1}$. Suppose $v_{2}, v_{2}^{\prime} \notin H_{i}$ for $i=1,2$. Let $T_{v_{2}}=T\left[H_{4}, X_{2}\right]$ and $T_{v_{2}^{\prime}}=T\left[H_{3}, X_{2}^{\prime}\right]$. By before exposed $X_{2}, X_{2}^{\prime} \neq H_{1}, H_{2}$. It is easy to prove that $X_{2}$ is good in $T\left[H_{3}, H_{2}, X_{2}^{\prime}\right]$ and $X_{2}^{\prime}$ is good in $T\left[H_{3}, H_{2}, X_{2}\right]$. Then $G$ has two asteroidal quadruple contradicting Theorem 2.

Suppose $T$ is not a star. Let $C_{1}$ and $C_{2}$ be the vertices of degree 3 in $T$. In order to fix ideas $H_{1}, H_{2} \in \operatorname{Bouq}\left(C_{1}\right)$ and $H_{3}, H_{4} \in \operatorname{Bouq}\left(C_{2}\right)$.

We will analyze two cases:

1. There is a vertex crossing $C_{1}$ in $T\left[H_{1}, H_{2}\right]$. Hence by Lemma 7 , there is a vertex crossing $C_{2}$ in $T\left[H_{3}, H_{4}\right]$. By Lemma 2, each vertex of branches has a crossing. It is clear that $T\left(a_{1}, a_{2}\right)$ and $T\left(a_{3}, a_{4}\right)$ are directed paths.

(a) $\left|V\left(T\left[H_{i}, H_{i+1}\right]\right)\right|=3$ for $i=1,3$. Therefore there are two vertices $x_{1} \in N\left[a_{1}\right] \cap N\left[a_{2}\right]$ and $x_{2} \in N\left[a_{3}\right] \cap N\left[a_{4}\right]$. Then $a_{i}$ and $a_{i+1}$ are linked by a special connection of Type 1 , for $i=1,3$. Therefore $a_{i}$ and $a_{i+1}$ are linked by a strong connection.

(b) $\left|V\left(T\left[H_{i}, H_{i+1}\right]\right)\right|>3$ for some $i \in\{1,3\}$, there is at least a branch with more than two vertices and by Lemma 5 , there are edges in this branch having dominated edges outside.

i. There is at least a branch whose edges has only dominated edges in $T\left[C_{1}, C_{2}\right]$. In order to fix ideas, $e_{3}^{\prime} \in T\left[C_{1}, C_{2}\right]$. Observe that if $\left|V\left(T\left[C^{\prime}, H_{4}\right]\right)\right|>2$ then $e_{4}^{\prime} \notin T\left[H_{1}, H_{2}, C_{2}\right]$ because $T$ is a $D V$-model. In this case, $e_{4}^{\prime} \in T\left[C_{2}, H_{3}\right]$ then, 
by Lemma 14, $a_{3}$ and $a_{4}$ are linked by a special connection of Type 1 or Type 2. Hence $a_{3}$ and $a_{4}$ are linked by a strong connection.

Suppose $\left|V\left(T\left[C_{2}, H_{4}\right]\right)\right|=2$. By Lemma 10 there is only one edge with label contained in $\operatorname{lab}\left(e_{3}^{\prime}\right)$ it is $e_{3}^{\prime}$ itself. Therefore $G \backslash \operatorname{lab}\left(e_{3}^{\prime}\right)$ has only two connected components, one containing $a_{1}, a_{2}$ and the other containing $a_{3}, a_{4}$.

Let $T_{1}$ be the connected component of $T-e_{3}^{\prime}$ containing $H_{1}, H_{2}$ and $T_{2}$ the other. As $e_{3}^{\prime}$ does not have dominated edges in $T\left[A_{3}^{\prime}, H_{3}, H_{4}\right]$, we have $B_{3}^{\prime}$ is always a leaf in each clique tree of $G_{T_{2}+e_{3}^{\prime}}$. Hence $l\left(G_{T_{2}+e_{3}^{\prime}}\right)=3$. Also if $A_{3}^{\prime} \neq C_{2}$ then it does not have dominated edges in $T\left[H_{3}, C_{2}\right]$, otherwise let $e=A B$ be a dominated edge by $A_{3}^{\prime}$ with $B \in T\left[A, C_{2}\right]$ and $T^{\prime}=T-\left\{e, e_{3}^{\prime}\right\}+B_{3}^{\prime} B+A_{3}^{\prime} A$ it is a $R D V$-model of $G$ rooted on $H_{1}$ or $H_{2}$, a contradiction.

Let $T^{\prime}$ be other $D V$-model of $G$. Let ${\widetilde{e^{\prime}}}_{3}$ be the edge of $T^{\prime}$ such that $\operatorname{lab}\left({\widetilde{e^{\prime}}}_{3}\right)=$ $l a b\left(e_{3}^{\prime}\right)$. It is clear from Lemma 10 and connectivity that if $T_{1}^{\prime}$ and $T_{2}^{\prime}$ are the two connected components of $T^{\prime}-\widetilde{e}_{3}^{\prime}$ then $V\left(T_{1}^{\prime}\right)=V\left(T_{1}\right)$ and $V\left(T_{2}^{\prime}\right)=V\left(T_{2}\right)$. On the other hand, there is $v \in V(G) \cap \operatorname{lab}\left(e_{3}^{\prime}\right)$ such that $V\left(T_{2}^{\prime}\right) \backslash\left\{H_{3}, H_{4}\right\} \subseteq$ $C_{v} \subseteq V\left(T_{2}^{\prime}\right) \backslash H_{4}$. Clearly, $\ln \left(T_{2}^{\prime}\right) \leq 4$. We choose $v$ maximizing $\left|C_{v}\right|$.

$-\ln \left(T_{2}^{\prime}\right)=4$ then $C_{v}=V\left(T_{2}^{\prime}\right) \backslash\left\{H_{3}, H_{4}\right\}$. On the other hand, by Lemma 3 , there are at least 4 vertices $v_{1}, v_{1}^{\prime}, v_{2}, v_{2}^{\prime}$ with $v_{i}^{\prime} \in H_{3}$ for $i=1,2$ and $v_{j} \in H_{4}$ for $j=1,2$ such that $\left|C_{v_{i}^{\prime}} \cap C_{v}\right| \geq 1,\left|C_{v_{j}} \cap C_{v}\right| \geq 1,\left|C_{v_{2}^{\prime}}\right|>\left|C_{v_{1}^{\prime}}\right|$ and $\left|C_{v_{2}}\right|>\left|C_{v_{1}}\right|$.

Now, if $T^{\prime}\left(a_{3}, a_{4}\right)$ is not a directed path of $T^{\prime}$ we must analyze the following 2 situations showed in Figures 2 and 3 according to the positions of $v_{2}$ and $v_{2}^{\prime}$. In both figures we draw only $T_{2}^{\prime}$ being $H_{3}, H_{4} ; B, A$ its leaves, $X, Y$ the vertices of degree 3 , may be the same, $Y \in T_{2}^{\prime}[B, X], \operatorname{lab}\left(e_{3}^{\prime}\right) \subset A$ and $l a b\left(e_{3}^{\prime}\right) \subset B$.
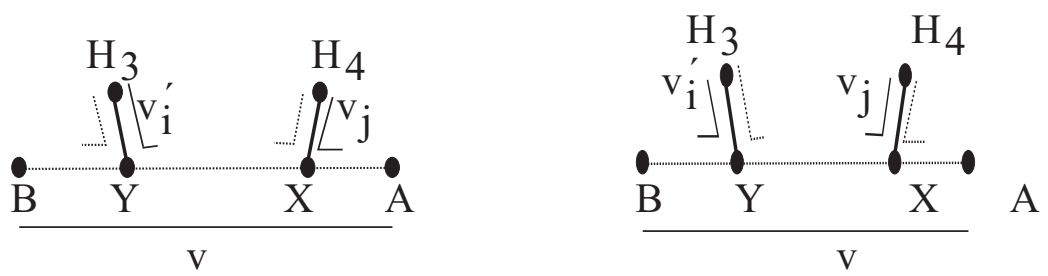

Fig. 2. $T_{2}^{\prime}$ is a proper subtree of $T^{\prime}$ a DV-model of $\mathrm{G}$

By the symmetry we can assume in Figure 2 that $v_{2}^{\prime}$ is a vertex crossing $Y$ in $T_{2}^{\prime}\left[H_{3}, A\right]$ and $v_{2}$ is a vertex crossing $X$ in $T_{2}^{\prime}\left[H_{4}, A\right]$. Hence $T_{2}^{\prime}$ rooted on $A$ is a $R D V$-model of $G_{T_{2}^{\prime}}$. We will denote this $R D V$-model by $T_{2}^{\prime A}$. Observe that $A$ contains $l a b\left(e_{3}^{\prime}\right)$. Hence $T_{1}+T_{2}^{\prime A}+A B_{3}^{\prime}$ is a $R D V$-model of $G$ rooted on $H_{1}$ if there is a vertex crossing $C_{1}$ in $T\left[H_{1}, C_{2}\right]$ or on $H_{2}$ if there is a vertex crossing $C_{1}$ in $T\left[H_{2}, C_{2}\right]$, a contradiction.

In Figure $3, v_{2}^{\prime}$ is a vertex crossing $Y$ in $T_{2}^{\prime}\left[H_{3}, B\right]$ and $v_{3}$ is a vertex crossing $X$ in $T_{2}^{\prime}\left[H_{4}, A\right]$.

If there is an edge $e=L M \in T_{2}^{\prime}[X, Y], M \in T_{2}^{\prime}[Y, L]$ and $l a b(e) \subseteq B$ then we build $T_{2}^{\prime \prime}=T_{2}^{\prime}-e+L B$ that is a $D V$-model of $G_{T_{2}^{\prime}}$ which can be rooted on $A$. Observe that we can conclude as in case of Figure 2, considering $T_{2}^{\prime \prime}$ instead of $T_{2}^{\prime}$. Therefore none edge of $T_{2}^{\prime}[X, Y]$ is dominated by $B$. Analogously, none is dominated by $A$. 


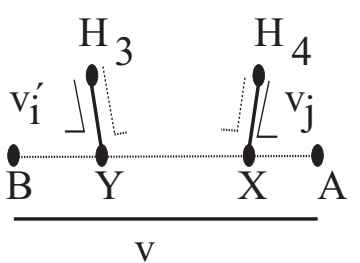

Fig. 3. $T_{2}^{\prime}$ is a proper subtree of $T^{\prime}$ a DV-model of $\mathrm{G}$

By before exposed if $A_{3}^{\prime} \neq C_{2}$ then $A=A_{3}^{\prime}$ or $B=A_{3}^{\prime}$. Suppose $A=$ $A_{3}^{\prime}$. Hence, by Property 2, $G_{T_{2}^{\prime}}$ has a $R D V$-model rooted on $B$. As before exposed $T_{1}+T_{2}^{\prime A}+A B_{3}^{\prime}$ is a $R D V$-model of $G$ rooted on $H_{1}$ if there is a vertex crossing $C_{1}$ in $T\left[H_{1}, C_{2}\right]$ or on $H_{2}$ if there is a vertex crossing $C_{1}$ in $T\left[H_{2}, C_{2}\right]$, a contradiction.

If $A_{3}^{\prime}=C_{2}$ then $A$ and $B$ are bad leaf, so both of them has dominated edges. As none edge of $T_{2}^{\prime}[X, Y]$ is dominated by $A$ or $B$ then dominated edges of $B$ are in $T_{2}^{\prime}[X, A]$ and dominated edges of $A$ are in $T_{2}^{\prime}[B, Y]$. Therefore, there are at least two edges one dominated by $A$ and the other dominated by $B$ with the same label. But the equivalent edges to these are in the same branch of $T_{2}$ contradicting the Lemma 4.

- $\ln \left(T_{2}^{\prime}\right)=3$ then $C_{v}=V\left(T_{2}^{\prime}\right) \backslash\left\{H_{4}\right\}$ or $C_{v}=V\left(T_{2}^{\prime}\right) \backslash\left\{H_{3}, H_{4}\right\}$. Let $B, H_{3}, H_{4}$ be the leaves of $T_{2}^{\prime}$ and $Y$ the vertex of degree 3 in $T_{2}^{\prime}$. We analyze the case in Figure 4 because the other is similar. (Remember that we assume $T^{\prime}\left(a_{3}, a_{4}\right)$ is not a directed path)

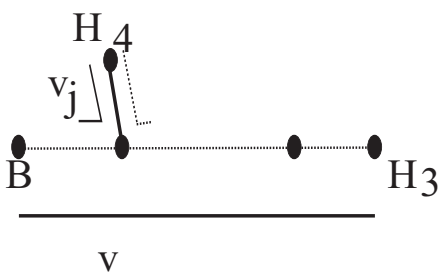

Fig. 4. $T_{2}^{\prime}$ is a proper subtree of $T^{\prime}$ a DV-model of $\mathrm{G}$

In case $v_{2}$ is a vertex crossing $Y$ in $T_{2}^{\prime}\left[H_{4}, B\right]$, since $T_{2}^{\prime}$ can be rooted on $B$ and $l a b\left(e_{3}^{\prime}\right) \subseteq B$ the tree $T_{1}+T_{2}^{\prime B}+A_{3}^{\prime} B$ is a $R D V$-model of $G$ rooted on $H_{1}$ if there is a vertex crossing $C_{1}$ in $T_{1}\left[H_{1}, C_{2}\right]$ or on $H_{2}$ if there is a vertex crossing $C_{1}$ in $T_{1}\left[H_{2}, C_{2}\right]$, a contradiction.

Therefore $a_{3}$ and $a_{4}$ are linked by a strong connection.

If $\left|V\left(T\left[H_{1}, H_{2}\right]\right)\right|=3$ then $a_{1}$ and $a_{2}$ are linked by a special connection of Type 1. Therefore, they are linked by a strong connection.

If $\left|V\left(T\left[H_{1}, H_{2}\right]\right)\right|>3$, suppose $\left|V\left(T\left[H_{1}, C_{1}\right]\right)\right|>2$ then by Lemma 5 , there is $e_{1}^{\prime}$ dominated edge by $e_{1}$.

If $e_{1}^{\prime} \in T\left[C_{1}, C_{2}\right]$, by the previous analysis taking $e_{1}^{\prime}$ instead of $e_{3}^{\prime}$, then $a_{1}$ and $a_{2}$ are linked by a strong connection.

In case $e_{1}^{\prime} \notin T\left[C_{1}, C_{2}\right]$, as $e_{3}^{\prime} \in T\left[C_{1}, C_{2}\right]$ and $T$ is a $D V$-model then $e_{1}^{\prime} \notin$ $T\left[C_{2}, H_{4}\right]$. By Lemma 10, $e_{3}^{\prime}$ does not dominate $e_{1}^{\prime}$ then $e_{1}^{\prime} \notin T\left[C_{2}, H_{3}\right]$. Hence $e_{1}^{\prime} \in T\left[H_{2}, C_{1}\right]$ and by Lemma $14, a_{1}$ and $a_{2}$ are linked by a special connection of Type 1 or Type 2 . Therefore they are linked by a strong connection. 
ii. None branch has edges whose dominated edges are in $T\left[C_{1}, C_{2}\right]$. If $e_{3}^{\prime} \in T\left[H_{4}, C_{2}\right]$ then, by Corollary $2, a_{3}$ and $a_{4}$ are linked by a special connection of Type 1 or Type 2. Therefore they are linked by a strong connection.

In case $\left|V\left(T\left[H_{1}, H_{2}\right]\right)\right|=3$ then $a_{1}$ and $a_{2}$ are linked by a special connection of Type 1. Therefore they are linked by a strong connection.

In case $\left|V\left(T\left[H_{1}, H_{2}\right]\right)\right|>3$, suppose $\left|V\left(T\left[H_{1}, C_{1}\right]\right)\right|>2$ then by Lemma 5 there is $e_{1}^{\prime}$ a dominated edge by $e_{1}$. By the type of connection between $a_{3}$ and $a_{4}$ it is clear that $e_{1}^{\prime} \notin T\left[H_{3}, H_{4}\right]$. Hence $e_{1}^{\prime} \in T\left[H_{2}, C_{1}\right]$ then, by Lemma $14, a_{1}$ and $a_{2}$ are linked by a special connection of Type 1 or Type 2. Therefore they are linked by a strong connection.

2. There is no vertex crossing $C_{1}$ in $T\left[H_{1}, H_{2}\right]$. Hence by Lemma 7 , there is no vertex crossing $C_{2}$ in $T\left[H_{3}, H_{4}\right]$. Also by Lemma 12 , ever vertex of $T\left[C_{1}, C_{2}\right]$ has a vertex crossing. Therefore $T\left(a_{1}, a_{3}\right), T\left(a_{1}, a_{4}\right), T\left(a_{2}, a_{3}\right)$ and $T\left(a_{2}, a_{4}\right)$ are directed paths.

(a) $\left|V\left(T\left[H_{i}, C_{1}\right]\right)\right|=\left|V\left(T\left[H_{j}, C_{2}\right]\right)\right|=2$ for some $i=1,2 ; j=3,4$. By symmetry, suppose $\left|V\left(T\left[H_{2}, C_{1}\right]\right)\right|=\left|V\left(T\left[H_{4}, C_{2}\right]\right)\right|=2$. Let $T^{\prime}$ be a $d v$-model of $G$. By Property $3, \ln \left(T^{\prime}\right)=4$. Let $X$ and $Y$ be vertices of $T^{\prime}$ with degree 3 .

i. $X, Y \in T^{\prime}\left[H_{1}, H_{2}\right]$. Clearly $X \in T^{\prime}\left[H_{1}, H_{3}\right]$ or $X \in T^{\prime}\left[H_{1}, H_{4}\right]$. By symmetry, suppose $X \in T^{\prime}\left[H_{1}, H_{3}\right]$. If there is a vertex crossing $X$ in $T^{\prime}\left[H_{1}, H_{3}\right]$ then by Lemma 7 there is a vertex crossing $Y$ in $T\left[H_{2}, H_{4}\right]$. Also by Lemma 2 every vertex of $T^{\prime}\left[H_{i}, X\right]$ for $i=1,3$ has a vertex crossing. Therefore $T^{\prime}\left(a_{1}, a_{3}\right)$ and $T^{\prime}\left(a_{2}, a_{4}\right)$ are directed paths. As $T\left(a_{1}, a_{3}\right)$ and $T\left(a_{2}, a_{4}\right)$ are directed paths then $a_{1}, a_{3}$ and $a_{2}, a_{4}$ are linked by a strong connection. If there is no vertex crossing $X$ in $T^{\prime}\left[H_{1}, H_{3}\right]$ then by Lemma 7 there is no vertex crossing $Y$ in $T\left[H_{2}, H_{4}\right]$. Also by Lemma 12, every vertex of $T^{\prime}[X, Y]$ has a vertex crossing. Therefore $T^{\prime}\left(a_{1}, a_{2}\right), T^{\prime}\left(a_{1}, a_{4}\right), T^{\prime}\left(a_{2}, a_{3}\right)$ and $T^{\prime}\left(a_{3}, a_{4}\right)$ are directed paths. As $T\left(a_{1}, a_{4}\right)$ and $T\left(a_{2}, a_{3}\right)$ are directed paths then $a_{1}, a_{4}$ and $a_{2}, a_{3}$ are linked by a strong connection.

ii. $X, Y \notin T^{\prime}\left[H_{1}, H_{2}\right]$. Then $X \in T^{\prime}\left[H_{1}, H_{2}\right]$ or $Y \in T^{\prime}\left[H_{1}, H_{2}\right]$. Suppose $X \in$ $T^{\prime}\left[H_{1}, H_{2}\right]$. If there is no a crossing $X$ in $T^{\prime}\left[H_{1}, H_{2}\right]$ then there is no vertex cross$\operatorname{ing} Y$ in $T^{\prime}\left[H_{3}, H_{4}\right]$. Therefore $T^{\prime}\left(a_{1}, a_{3}\right), T^{\prime}\left(a_{1}, a_{4}\right), T^{\prime}\left(a_{2}, a_{3}\right)$ and $T^{\prime}\left(a_{2}, a_{4}\right)$ are directed paths. As $T\left(a_{1}, a_{3}\right)$ and $T\left(a_{2}, a_{4}\right)$ are directed paths then $a_{1}, a_{3}$ and $a_{2}, a_{4}$ are linked by a strong connection. If there is a crossing $X$ in $T^{\prime}\left[H_{1}, H_{2}\right]$ then by Lemma 7 there is a vertex crossing $Y$ in $T^{\prime}\left[H_{3}, H_{4}\right]$. As $T\left(a_{1}, a_{2}\right)$ and $T\left(a_{3}, a_{4}\right)$ are not directed paths then $a_{i}$ and $a_{i+1}$ for $i=1,3$ are not linked by a special connection of Type 1 . Hence $\left|V\left(T^{\prime}\left[H_{i}, H_{i+1}\right]\right)\right|>3$ for $i=1$, 3. Observe that if $\left|V\left(T^{\prime}\left[H_{i}, X\right]\right)\right| \geq 3$ for $i=1,2$ then there exist $e_{i} \in T^{\prime}\left(H_{i}, X\right]$ having a dominated edge $e_{i}^{\prime}$ for $i=1,2$. As $G$ is a $D V$ graph $e_{1}^{\prime}$ and $e_{2}^{\prime}$ do not be both in $T^{\prime}\left[X, H_{3}, H_{4}\right]$. Hence at least one of them is in $T^{\prime}\left[H_{1}, H_{2}\right]$ and by Lemma 14, there is a special connection of Type 1 or 2 linking $a_{1}, a_{2}$ a contradiction because there is no vertex crossing $C_{1}$ in $T\left[H_{1}, H_{2}\right]$. Then $H_{1} X \in E\left(T^{\prime}\right)$ or $H_{2} X \in E\left(T^{\prime}\right)$ and analogously $H_{3} Y \in E\left(T^{\prime}\right)$ or $H_{4} Y \in E\left(T^{\prime}\right)$. By symmetry suppose that $H_{2} X$ and $H_{4} Y$ are edges of $T^{\prime}$. Hence exist $e_{1}^{\prime}$ and $e_{3}^{\prime}$.

If $e_{1}^{\prime} \in T^{\prime}[X, Y]$ then we build a $R D V$-model with $T^{\prime}$ and $T$. More clearly, as $e_{1}^{\prime} \in T^{\prime}[X, Y]$ by Lemma $10, e_{1}^{\prime}$ does not have a dominated edge in $T^{\prime}$. Hence $G \backslash l a b\left(e_{1}^{\prime}\right)$ has only two connected component. Let $T_{1}^{\prime}$ and $T_{2}^{\prime}$ the connected component of $T^{\prime}-e_{1}^{\prime}$. Let $\widetilde{e_{1}^{\prime}}$ be the equivalent edge to $e_{1}^{\prime}$ in $T$ and $T_{1}, T_{2}$ the connected component of $T-\widetilde{e_{1}^{\prime}}$. By before exposed $V\left(T_{i}^{\prime}\right)=V\left(T_{i}\right)$ for $i=1,2$. 
Let $\widetilde{e_{1}^{\prime}}=\widetilde{A_{1}} \widetilde{B_{1}}$ and $e_{1}^{\prime}=A_{1}^{\prime} B_{1}^{\prime}$ with $\widetilde{B_{1}} \in T^{\prime}\left[X, \widetilde{A_{1}}\right]$ and $B_{1}^{\prime} \in T\left[C_{1}, A_{1}\right]$. We build $T_{1}+B_{1}^{\prime} \widetilde{A_{1}}+T_{2}^{\prime}$; it is a $R D V$-model of $G$, a contradiction. Analogously if $e_{3}^{\prime} \in T^{\prime}[X, Y]$. Hence $e_{1}^{\prime} \notin T^{\prime}[X, Y]$ and $e_{3}^{\prime} \notin T^{\prime}[X, Y]$. Then the only possibility is $e_{1}^{\prime}=Y H_{4}$ and $e_{2}^{\prime}=X H_{2}$ but this contradicts that $G$ is $D V$.

If $e_{1}^{\prime} \in T^{\prime}\left[H_{3}, Y\right]$ and $e_{3}^{\prime} \in T^{\prime}\left[H_{1}, X\right]$ then every edge $e \in T^{\prime}[X, Y]$ dominates $e_{1}^{\prime}$ and $e_{3}^{\prime}$ contradicting Lemma 11.

(b) We assume that (a) does not hold then if $\left|V\left(T\left[H_{1}, C_{1}\right]\right)\right|=2$ or $\left|V\left(T\left[H_{2}, C_{1}\right]\right)\right|=2$ then $\left|V\left(T\left[H_{3}, C_{2}\right]\right)\right|>2$ and $\left|V\left(T\left[H_{4}, C_{2}\right]\right)\right|>2$.

As there is no vertex crossing $T\left[H_{3}, H_{4}\right]$ then $e_{3}^{\prime} \notin T\left[H_{4}, C_{2}\right]$ and $e_{4}^{\prime} \notin T\left[H_{3}, C_{2}\right]$. As $T$ is a $D V$-model then $e_{3}^{\prime} \in T\left[H_{1}, C_{1}\right]$ and $e_{4}^{\prime} \in T\left[H_{2}, C_{1}\right]$ or viceversa. In both case there are a special connection of Type 1 or 2 between $a_{1}, a_{3}$ and $a_{2}, a_{4}$ or $a_{1}, a_{4}$ and $a_{2}, a_{3}$.

\section{Concluding remarks}

The celebrate characterization of interval graph given by Lekkerkerker and Boland, related chordal non interval graph with asteroidal triples. This kind of characterization is given by Cameron, Hoáng and Lévêque for chordal non DV graph. Analyzing the difference between the nested classes, we proved [4] that UV non DV graphs always contains an odd sun that is an odd asteroidal with forced path between consecutives vertices. Since as we shown before DV non RDV graph must have an asteroidal quadruple it is natural think if some type of quadruple must appear with strong paths not allowed that any DV-model could be rooted. Althout we can not describe totally the special connections that force the direction in any DV-models, we prove that every DV minimally non RDV graph must have an asteroidal quadruple with two pairs of strong paths when the leafage is four. In future work, we will try to complete the type of those paths.

\section{References}

1. K. Cameron, C. T. Hoáng, B. Lévêque. Asteroids in rooted and directed path graphs. Electronic Notes in Discrete Mathematics 32 (2009) 67-74.

2. K. Cameron, C. T. Hoáng, B. Lévêque. Characterizing directed path graphs by forbidden asteroids. Journal of Graph Theory 68 (2011) 103-112.

3. S. Chaplick, J. Stacho, The vertex leafage of chordal graphs, arXiv:1104.2524v2, manuscript 2012.

4. S. Chaplick, M. Gutierrez, B. Lévêque, S. B. Tondato, From path graphs to directed path graphs, WG'10, Lecture Notes in Computer Science 6410 (2010) 256-265.

5. F. Gavril. The intersection graphs of subtrees in trees are exactly the chordal graphs. $J$. Combin. Theory B 16 (1974) 47-56.

6. M. Gutierrez, B. Lévêque, S. B. Tondato, Asteroidal quadruples in non rooted path graphs, manuscript 2012.

7. M. Gutierrez, S. B. Tondato, On path models of path graphs, manuscript 2011.

8. C. G. Lekkerkerker, J. Ch. Boland, Representation of finite graph by a set of intervals on the real line, Fundamenta Mathematicae Li (1962) 45-64. 
9. B. Lévêque, F. Maffray, M. Preissmann, Characterizing path graphs by forbidden induced subgraphs, Journal of Graph Theory 62 (2009) 369-384.

10. I. Lin, T. McKee and D. B. West, The leafage of a chordal graphs, Discussiones Mathematicae, Graph Theory 18 (1998) 23-48.

11. C. Monma, V. Wei, Intersection graphs of paths in a tree, J. Combin. Theory B 41 (1986) $141-181$.

12. B. S. Panda, The forbidden subgraph characterization of directed vertex graphs, Discrete Mathematics 196 (1999) 239-256. 